\title{
Transthoracic coronary flow reserve and dobutamine derived myocardial function: a 6-month evaluation after successful coronary angioplasty
}

\author{
Silvana Cicala ${ }^{1}$, Maurizio Galderisi*1, Pasquale Guarini², \\ Arcangelo D'Errico' ${ }^{1}$ Pasquale Innelli ${ }^{1}$, Moira Pardo ${ }^{1}$, \\ Giancarlo Scognamiglio ${ }^{2}$ and Oreste de Divitiis ${ }^{1}$
}

\begin{abstract}
Address: ${ }^{1}$ Cardioangiology Unit, Department of Clinical and Experimental Medicine, Federico II University Hospital Naples, Italy and ${ }^{2}$ Division of Cardiology, "Villa dei Fiori" Hospital Naples, Italy

Email: Silvana Cicala - s.cicala1@virgilio.it; Maurizio Galderisi* - mgalderi@unina.it; Pasquale Guarini - uticacerra@libero.it; Arcangelo D'Errico - axcangio@libero.it; Pasquale Innelli - pasquale.innelli@libero.it; Moira Pardo - moira.pardo@libero.it; Giancarlo Scognamiglio - gscogl@tiscalinet.it; Oreste de Divitiis - devitiis@unina.it

* Corresponding author
\end{abstract}

Published: 06 December 2004

Cardiovascular Ultrasound 2004, 2:26 doi:10.1186/1476-7120-2-26

This article is available from: http://www.cardiovascularultrasound.com/content/2/1/26

(C) 2004 Cicala et al; licensee BioMed Central Ltd.

This is an Open Access article distributed under the terms of the Creative Commons Attribution License (http://creativecommons.org/licenses/by/2.0), which permits unrestricted use, distribution, and reproduction in any medium, provided the original work is properly cited.
Received: 04 November 2004

Accepted: 06 December 2004

\begin{abstract}
After percutaneous transluminal coronary angioplasty (PTCA), stress-echocardiography and gated single photon emission computerized tomography (g-SPECT) are usually performed but both tools have technical limitations. The present study evaluated results of PTCA of left anterior descending artery (LAD) six months after PTCA, by combining transthoracic Doppler coronary flow reserve (CFR) and color Tissue Doppler (C-TD) dobutamine stress.

Six months after PTCA of LAD, 24 men, free of angiographic evidence of restenosis, underwent standard Doppler-echocardiography, transthoracic CFR of distal LAD (hyperemic to basal diastolic coronary flow ratio) and C-TD at rest and during dobutamine stress to quantify myocardial systolic $\left(S_{m}\right)$ and diastolic $\left(E_{m}\right.$ and $A_{m}, E_{m} / A_{m}$ ratio) peak velocities in middle posterior septum. Patients with myocardial infarction, coronary stenosis of non-LAD territory and heart failure were excluded. According to dipyridamole g-SPECT, I3 patients had normal perfusion and II with perfusion defects. The 2 groups were comparable for age, wall motion score index (WMSI) and C-TD at rest. However, patients with perfusion defects had lower CFR $(2.11 \pm 0.4$ versus $2.87 \pm 0.6, p<0.002)$ and septal $S_{m}$ at high-dose dobutamine $(p<0.01)$, with higher WMSI $(p<0.05)$ and stress-echo positivity of LAD territory in $5 / I$ I patients. In the overall population, CFR was related negatively to high-dobutamine WMSI $(r=-0.50, \mathrm{p}<0.0 \mathrm{I})$ and positively to high-dobutamine $\mathrm{S}_{\mathrm{m}}$ of middle septum $(r=0.55, p<0.005)$.

In conclusion, even in absence of epicardial coronary restenosis, stress perfusion imaging reflects a physiologic impairment in coronary microcirculation function whose magnitude is associated with the degree of regional functional impairment detectable by C-TD.
\end{abstract}




\section{Introduction}

Percutaneous transluminal coronary angioplasty (PTCA) has deeply modified the effective management of coronary artery disease [1]. Coronary artery restenosis is unfrequent when PTCA is associated to coronary stenting application which is able to enlarge the lumen area stenosis $[2,3]$. However, even in absence of coronary artery restenosis, the results of revascularization can be suboptimal because of coronary microvessel dysfunction subsequent to the procedure $[4,5]$. This issue may be intriguing for management of patients undergone PTCA.

The non-invasive assessment after PTCA is usually performed by gated single photon emission computerized tomography (g-SPECT) [6,7] and by stress echocardiography $[7,8]$. However, both these tools present technical limitations, g-SPECT having a low specificity [9] and semi-quantitative echocardiographic wall motion analysis low sensitivity [10].

In the last years, great interest has been developed about new echocardiographic techniques as Doppler-derived coronary flow reserve (CFR) $[11,12]$ and color Tissue Doppler (C-TD) $[13,14]$. The first tool provides reliable information about coronary microvascular function in absence of epicardial coronary stenosis [15] while C-TD is able to quantify left ventricular (LV) myocardial performance both at rest and during pharmacological stress $[13,14]$.

On these grounds, aim of the present study was to assess C-TD derived myocardial performance, both at rest and during pharmacologic stress, in relation to the function of coronary microcirculation determined by non invasive CFR after successful PTCA of LAD.

\section{Methods}

\section{Study population}

Among 30 patients who had undergone PTCA with stenting for significant LAD stenosis between September and October 2000, 24 patients (age $=50-64$ years) free of coronary angiographic evidence of LAD restenosis 6 months after the procedure, entered the study and performed noninvasive test screening in the same period of the invasive assessment ( \pm 7 days). The informed consent of all patients and approval of Institutional Committee were obtained. Patients were excluded for acute and previous myocardial infarction (according to ECG at rest), concomitant coronary stenosis of right coronary artery and/or circumflex artery, congestive heart failure, valvular heart disease, primary cardiomyopathy, atrial fibrillation, inadequate quality echocardiograms. On the basis of g-SPECT dipyridamole induced perfusion defects, the study population was divided into 2 groups: without and with perfusion defects.

\section{Procedures}

Patients underwent dipyridamole gated myocardial perfusion g-SPECT acquisition, transthoracic echocardiography, C-TD (both at baseline and during dobutamine stress) and non-invasive CFR determination by dipyridamole test. All echocardiographic measurements were analyzed without knowledge of the clinical data. According to the rules of the Institutional Committees, all patients withdrew cardiac drugs at least 2 days before the performance of the non invasive assessment.

\section{Dipyridamole g-SPECT}

Single day rest/dipyridamole g-SPECT was performed according to the standard methods by injecting patients

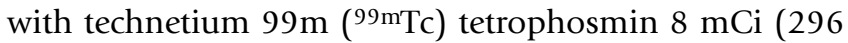
$\mathrm{MBq})$ at rest and $24 \mathrm{mCi}(888 \mathrm{MBq})$ after dipyridamole infusion by volumetric pump (dose of $0.14 \mathrm{mg} / \mathrm{kg} / \mathrm{min}$ in 4 minutes) $[16,17]$. A single stress SPECT corresponds to a dose exposure of about 500 chest x-ray. Qualitative assessment of reconstructed gated images was obtained on mid-short axis slices, vertical and horizontal long axis slices.

\section{Transthoracic Echocardiography}

Standard echocardiographic examinations were performed using a System FiVe, Vingmed Sound AB machine (GE, Horten, Norway), by a $2.5 \mathrm{MHz}$ transducer equipped with second harmonic capability. M-mode echocardiographic analysis was performed according to the criteria of the American Society of Echocardiography $[18]$ and LV mass indexed for height powered to 2.5 [19]. LV enddiastolic and end-systolic volumes were estimated according to the Simpson method [20] and LV ejection fraction derived.

\section{Stress protocols}

Dobutamine stress protocol was performed according to the standard method [21] using low and high-dose (up to $40 \mu \mathrm{g} / \mathrm{Kg} / \mathrm{min}$ ) by using the System FiVe Vingmed machine. C-TD of posterior septum was recorded at rest and during each dobutamine stage. CFR assessment was performed by HDI 5000 ultrasound machine (ATL Ultrasound, Bothell, Washington, USA), using a high-frequency $(7 \mathrm{MHz})$ transducer. The visualization of the distal portion of the left anterior descending artery and the recording of PW-Doppler derived coronary blood flow velocities performed at baseline and after dipyridamole infusion $(0.56 \mathrm{mg} / \mathrm{kg}$ over 4 minutes) according to the standards of our laboratory [14]. Blood pressure and a 12 lead ECG were recorded at rest and at the end of each stage of both dobutamine and dipyridamole tests.

\section{C-TD dobutamine stress echocardiography analysis}

Echocardiographic images were recorded on S-VHS videotapes and digitally stored on magneto-optical disk for 
subsequent analysis. Images were evaluated by 2 experienced observers. Baseline and stress wall motion analysis was performed by 2 experienced readers blinded to the other data. Regional wall motion was assessed with a 16segment model of the left ventricle and semiquantitatively graded from 1 to 4 as follows: $1=$ normal; $2=$ hypokinesia; 3 = akinesia; and $4=$ dyskinesia. A wall motion score index (WMSI), obtained dividing the sum of each segment scores by the number of the segments, was assessed both at baseline and at high-dose dobutamine.

C-TD acquisition of posterior septal wall was performed in real time, superimposed on 2-D images, at baseline and at the end of each dobutamine infusion stage. C-TD imaging was stored in digital format and analyzed off line on cine-loop as previously described [14]. The region of interest was the middle segment of the posterior septum where myocardial velocity profile was obtained. The middle posterior septum for measurements of C-TD was chosen since the perfusion of this myocardial segment is provided by a branch of LAD, where also CFR was determined. The reproducibility of C-TD of our laboratory has been reported, the intra- and inter-observer variability being $<3 \%$ and $<6 \%$ for all the measurements both at rest and at high-dose dobutamine [22].

\section{CFR Analysis}

Methods and reproducibility (intra-observer and interobserver variability $1.9 \%$ and $4.2 \%$ respectively) of our laboratory in measuring coronary blood flow reserve has been described [22]. By placing sample volume on the color signal, spectral Doppler of LAD flow showed the characteristic biphasic flow pattern with a larger diastolic and a smaller systolic component. Diastolic peak velocities were measured at baseline and after dipyridamole, by averaging the highest 3 spectral Doppler signals for each measurement. CFR was defined as the ratio of hyperemic to basal diastolic peak velocities. All images were recorded on a magneto-optical disk and analyzed off-line by 2 independent observers, blinded to the other data.

\section{Statistical Analysis}

The analyses were performed by SPSS for Windows release 8.0 (Chicago, Illinois, USA). Data are presented as mean value $\pm \mathrm{SD}$. Analysis of variance was used to assess intergroup differences. Linear regression analyses and partial correlation test was done using Pearson's method. Differences were considered significant at $\mathrm{p}<0.05$.

\section{Results}

\section{Characteristics of the study population}

The characteristics of the study population and both heart rate and blood pressure at baseline and at high-dose dobutamine are listed in Table 1 . The 2 groups were comparable for heart rate and blood pressure values both at rest and at stress dobutamine peak. Of note, the prevalence of arterial hypertension, diabetes mellitus, hypercholesterolemia and smoke was not different between groups and no patients of both groups presented g-SPECT derived myocardial perfusion defects at rest (data not reported in Table).

\section{Echocardiographic analysis}

The comparisons of echocardiographic measurements and CFR between the 2 groups are reported in Table 2 . Because of higher septal and posterior wall thickness, patients with myocardial perfusion defects after PTCA had greater LV mass index $(\mathrm{p}<0.05)$. LV ejection fraction was comparable between the 2 groups.

\section{Dobutamine test and Color TD analysis}

WMSI was comparable between the 2 groups at baseline $(1.07 \pm 0.10$ versus $1.15 \pm 0.11)$ whereas it was higher at low-dose dobutamine $(1.07 \pm 0.11$ versus $1.17 \pm 0.12)$ and at high-dose dobutamine $(1.07 \pm 0.12$ versus $1.20 \pm$ 0.14 ) (both $\mathrm{p}<0.05$ ) in patients with SPECT-derived perfusion defects than in controls. Positive dobutamine

Table I: Characteristics of the study population

\begin{tabular}{|c|c|c|c|}
\hline Variable & Normal Perfusion $n=13$ & Perfusion Defect $n=11$ & $\mathbf{P}$ \\
\hline Age (years) & $55.9 \pm 4.1$ & $58.4 \pm 3.1$ & NS \\
\hline Body mass index $\left(\mathrm{Kg} / \mathrm{m}^{2}\right)$ & $26.1 \pm 1.1$ & $26.3 \pm 0.8$ & NS \\
\hline Baseline Systolic BP (mm Hg) & $147.0 \pm 7.5$ & $149.2 \pm 11.6$ & NS \\
\hline Baseline Diastolic BP (mm Hg) & $85.1 \pm 7.5$ & $85.5 \pm 9.1$ & NS \\
\hline Baseline Heart rate (bpm) & $74.8 \pm 5.9$ & $73.7 \pm 6.9$ & NS \\
\hline DOB Systolic BP (mm Hg) & $151.1 \pm 7.5$ & $151.9 \pm 10.2$ & NS \\
\hline DOB Diastolic BP (mm Hg) & $83.2 \pm 6.3$ & $83.4 \pm 7.3$ & NS \\
\hline DOB Heart rate (bpm) & $139.6 \pm 5.4$ & $|4| .0 \pm 5.5$ & NS \\
\hline
\end{tabular}

$\mathrm{BP}=$ Blood Pressure, $\mathrm{DOB}=$ Dobutamine 
Table 2: Standard Doppler echocardiographic and CFR analysis

\begin{tabular}{|c|c|c|c|}
\hline Variable & Normal Perfusion & Perfusion Defect & $\mathbf{P}$ \\
\hline Septal wall thickness (mm) & $10.1 \pm 1.4$ & $11.2 \pm 0.4$ & $<0.02$ \\
\hline Posterior wall thickness (mm) & $10.2 \pm 1.4$ & $10.6 \pm 0.5$ & NS \\
\hline LV internal diastolic diameter (mm) & $54.7 \pm 2.6$ & $56.7 \pm 3.5$ & NS \\
\hline LV internal systolic diameter (mm) & $39.2 \pm 2.8$ & $39.6 \pm 2.9$ & NS \\
\hline 2-D Ejection Fraction (\%) & $54.8 \pm 6.0$ & $54.9 \pm 3.4$ & NS \\
\hline LV mass index $\left(\mathrm{g} / \mathrm{m}^{2.7}\right)$ & $49.6 \pm 10.7$ & $57.9 \pm 8.0$ & $<0.05$ \\
\hline
\end{tabular}

$\mathrm{LV}=$ left ventricular

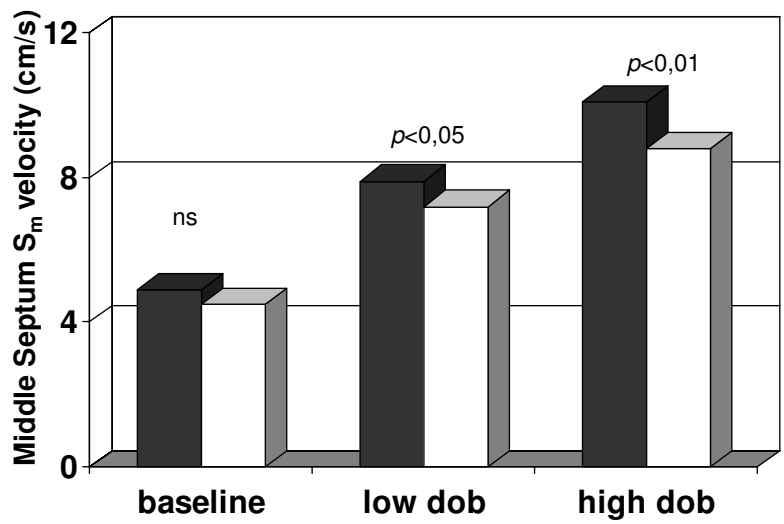

Normal Perfusion $(n=13)$

Perfusion Defect $(n=11)$

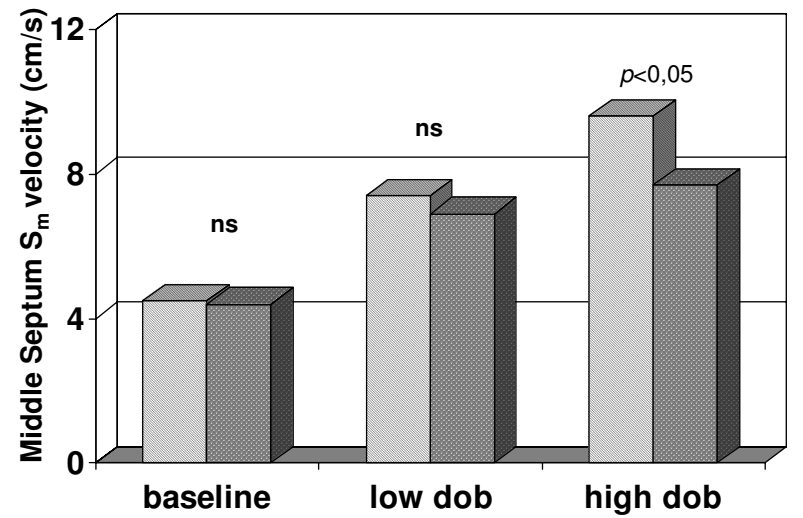

Dob normal wall motion $(n=6)$

Dob induced wall motion abnormalities $(n=5)$

\section{Figure I}

In the left panel comparison of $\mathrm{S}_{\mathrm{m}}$ peak velocity of middle posterior septum of patients without and with SPECT perfusion defects at rest, at low and at high-dose dobutamine. In the right panel comparison of $S_{m}$ peak velocity of middle posterior septum during dobutamine stress echocardiography in patients with perfusion defects having or not dobutamine-induced wall motion abnormalities. 
stress-echo involving LAD territory (and in particular midseptal region) was observed in $5 / 11$ patients $(45.4 \%)$ with SPECT perfusion defects.

C-TD diastolic measurements of mid-septum $\left(\mathrm{E}_{\mathrm{m}^{\prime}}, \mathrm{A}_{\mathrm{m}}, \mathrm{E}_{\mathrm{m}} /\right.$ $A_{m}$ ratio) were similar between the two groups at rest $\left(\mathrm{E}_{\mathrm{m}} /\right.$ $A_{m}$ ratio $=1.04 \pm 0.1$ and $1.03 \pm 0.3$ in patients with and without perfusion defects respectively, NS) and at low dose dobutamine $\left(\mathrm{E}_{\mathrm{m}} / \mathrm{A}_{\mathrm{m}}\right.$ ratio $=1.00 \pm 0.1$ and $1.13 \pm 0.4$ respectively, NS) while $E_{m} / A_{m}$ ratio was mildly different at high-dose dobutamine $(0.83 \pm 0.2$ and $0.70 \pm 0.2$ respectively, $\mathrm{p}<0.05)$. $S_{\mathrm{m}}$ peak velocities were lower in patients with perfusion defects at low- $(\mathrm{p}<0.05)$ and at high-dose dobutamine $(\mathrm{p}<0.01)$ and were significantly lower also in patients with perfusion defects showing stress induced wall motion abnormalities in comparison with patients with perfusion defects but no change of wall motion during dobutamine infusion (Figure 1).

\section{CFR analysis}

Coronary diastolic peak velocities were similar at rest between the two groups $(21.5 \pm 5.2 \mathrm{~cm} / \mathrm{s}$ in patients without perfusion defects and $22.0 \pm 19 \mathrm{~cm} / \mathrm{s}$ in patients with perfusion defects, NS) but significantly different after dipyridamole $(60.0 \pm 16.1 \mathrm{~cm} / \mathrm{s}$ versus $49.1 \pm 10.8 \mathrm{~cm} / \mathrm{s}$ respectively, $\mathrm{p}<0.05)$. Thus, CFR was $2.87 \pm 0.6$ in patients without defects and $2.11 \pm 0.4$ in patients with perfusion defects $(\mathrm{p}<0.002)$. Of note, analyzing the group with SPECT-derived perfusion defects, the patients with stress inducible wall motion abnormalities had lower CFR $(1.91 \pm 0.1)$ than those without change of WMSI during dobutamine infusion $(2.28 \pm 0.4)(\mathrm{p}=$ $0.06)$.

\section{Relationship between CFR and Dobutamine stress measurements} In the overall population, CFR was negatively related to WMSI at low-dose $(r=-0.46, p<0.02)$ and high-dose dobutamine $(\mathrm{r}=-0.50, \mathrm{p}<0.01)$. Among C-TD Doppler measurements, CFR was positively related to $S_{m}$ peak velocity of middle septum at low dose $(r=0.39, \mathrm{p}<0.05)$ and high-dose dobutamine $(\mathrm{r}=0.55, \mathrm{p}<0.0005)$ (Figure 2) while the relation of $S_{m}$ at baseline $(r=0.12)$ did not achieve the statistical significance. No relation of CFR was found with C-TD diastolic measurements of middle septum at any stage of dobutamine stress. Figure 3 shows a patient with SPECT derived normal perfusion: CFR is $>2$ and middle septal $S_{m}$ peak velocity has a significant increment from baseline to high-dose dobutamine $(\Delta=+9)$. Figure 4 displays a patient with a perfusion defect: CFR is reduced and middle septal $S_{m}$ increase from baseline to high-dose dobutamine is lower $(\Delta=+5)$.

\section{Discussion}

The present study used new ultrasound tools, as off-line quantitative C-TD $[13,14]$ and Doppler-derived CFR [15],

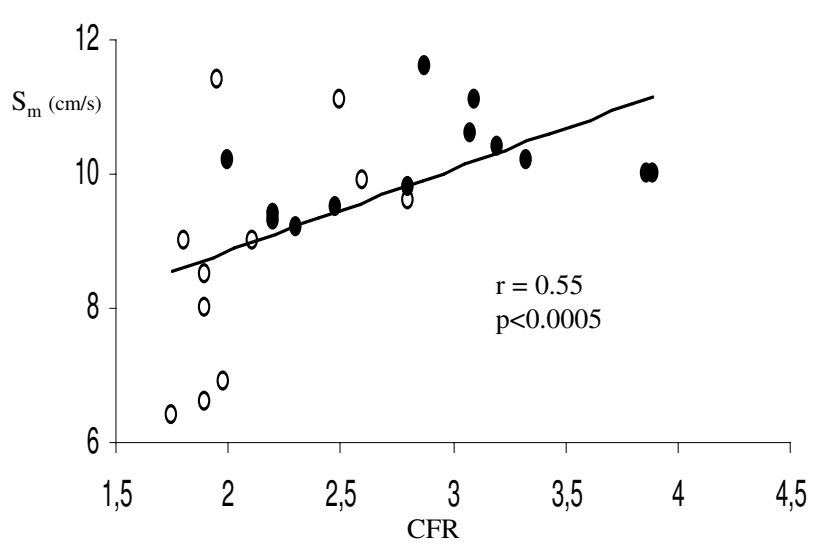

\section{Figure 2}

Positive association between CFR and C-TD derived $S_{m}$ peak velocity of middle septum at high-dose dobutamine. Full circles indicate patients with SPECT-derived myocardial perfusion defects; empty circles indicates patients without perfusion defects.

to evaluate long-term effects of PTCA on the relation between myocardial performance and coronary microvascular function, in the absence of angiographic coronary artery restenosis. According to dipyridamole g-SPECT, the population was divided into 2 groups, the first without and the second one with perfusion defect in the LAD territory, comparable for resting LV ejection fraction. Our findings show that, six months after successful PTCA of LAD, patients with myocardial perfusion defects present both lower CFR and reduced peak dobutamine myocardial systolic function of wall involved by LAD perfusion (i.e., middle posterior septum) in comparison with the control group and that CFR is positively related to stress peak $\mathrm{S}_{\mathrm{m}}$ velocity measured at middle septum in the overall population.

\section{CFR and perfusion defect after PTCA}

According to the study design, we intentionally selected patients without angiographic evidence of post-PTCA LAD restenosis. Worthy of note, the incidence of coronary restenosis was very low 6 months after the procedure, in accord with previous experiences about the combined use of stenting and PTCA [23]. Nevertheless, 11 our patients without restenosis showed dipyridamole g-SPECT LAD perfusion defects. Myocardial hypoperfusion may occur in patients without overt epicardial coronary artery stenosis having coronary microvessel damage $[24,25]$ and coronary microvascular dysfunction corresponds to a reduced CFR in the absence of epicardial coronary stenosis [26]. Accordingly, the patients of the present study 

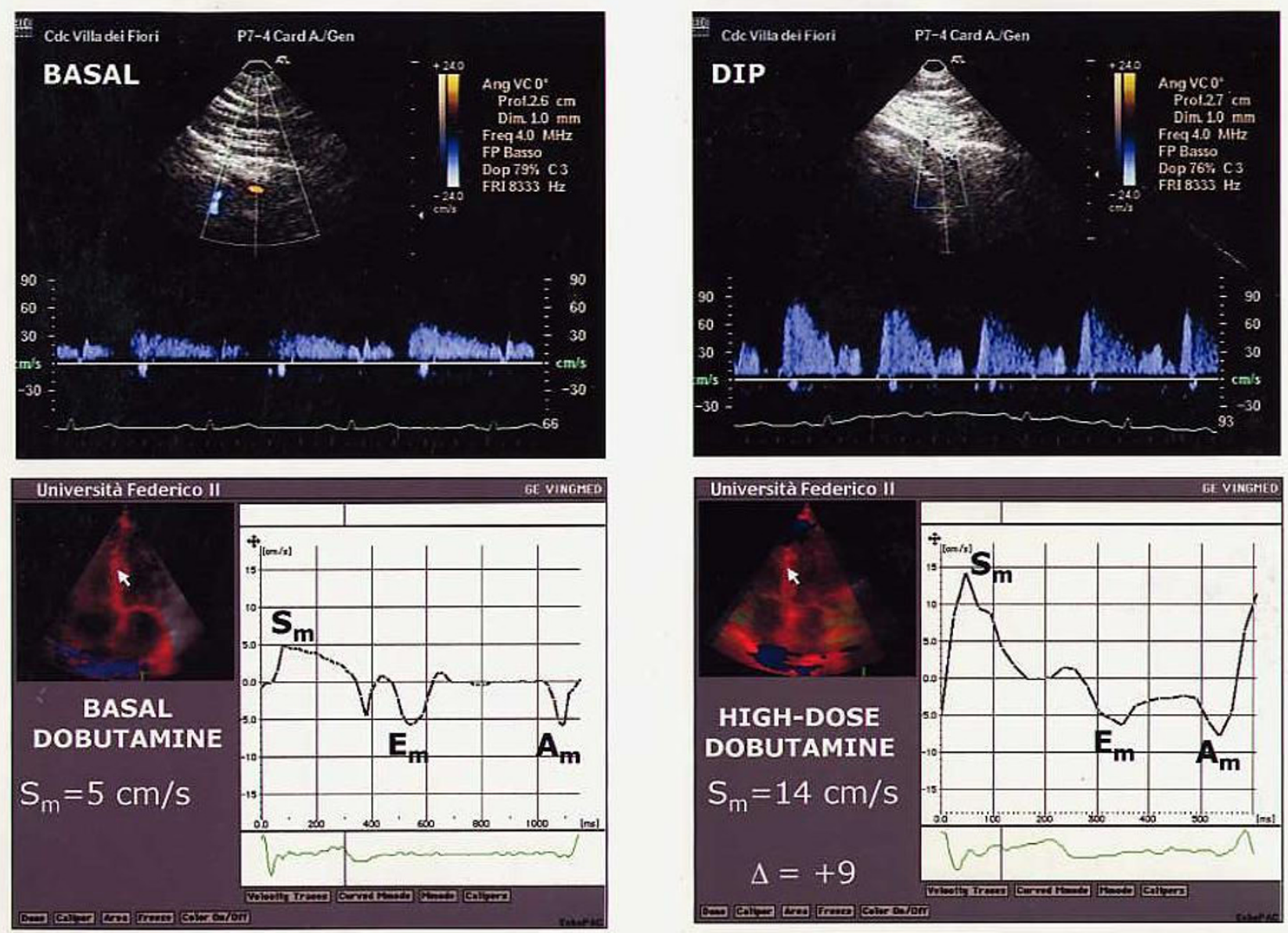

Figure 3

CFR and $\mathrm{S}_{\mathrm{m}}$ peak velocity of middle septum at high-dose dobutamine in a patient with SPECT derived normal perfusion. The upper panels show coronary artery flow velocity in the LAD at baseline and with a normal increase with dipyridamole (DIP). In the lower panels, myocardial systolic velocity $\left(S_{m}\right)$ shows a normal increase at high-dose dobutamine.

with long-standing SPECT perfusion defects showed lower CFR than the control group. An abnormal CFR had been already described immediately after balloon angioplasty $[5,27,28]$, probably because of a slow recovery of autoregulation in the microvascular bed [29]. This reduction is primarily due to an increased flow velocity at rest $[5,27,28]$, in relation to the failure of microvessel bed to vasoconstrict appropriately and/or to epicardial vasoconstriction mediated by a myogenic response and/or neural mechanism [30]. In contrast to previous studies showing normalization of CFR after three [31], five [32] or six months [5], CFR was persistently reduced in our patients with SPECT-derived perfusion defects. A suboptimal Doppler flow wire derived CFR had been observed six months after PTCA without restenosis by DEBATE investigators
[33]. In the suboptimal CFR group the reduction of CFR was mainly due to a long-standing elevation in resting peak velocities while in our patients with perfusion defects it was due to a blunted maximal vasodilator response to dypiridamole. It is conceivable that this alteration could depend on endothelial damage of coronary microcirculation [34] preceding the procedure and persisting long time after PTCA. Coronary microcirculatory vasoconstriction induced by endothelial dysfunction has been described as effect of spontaneous myocardial ischemia [35] as well as in conditions other than epicardial coronary artery stenosis, as diabetes mellitus [36,37] arterial hypertension [26,38] and LV hypertrophy $[39,40]$, which can alter microvascular function. However, an alternative interpretation of our findings include the pos- 

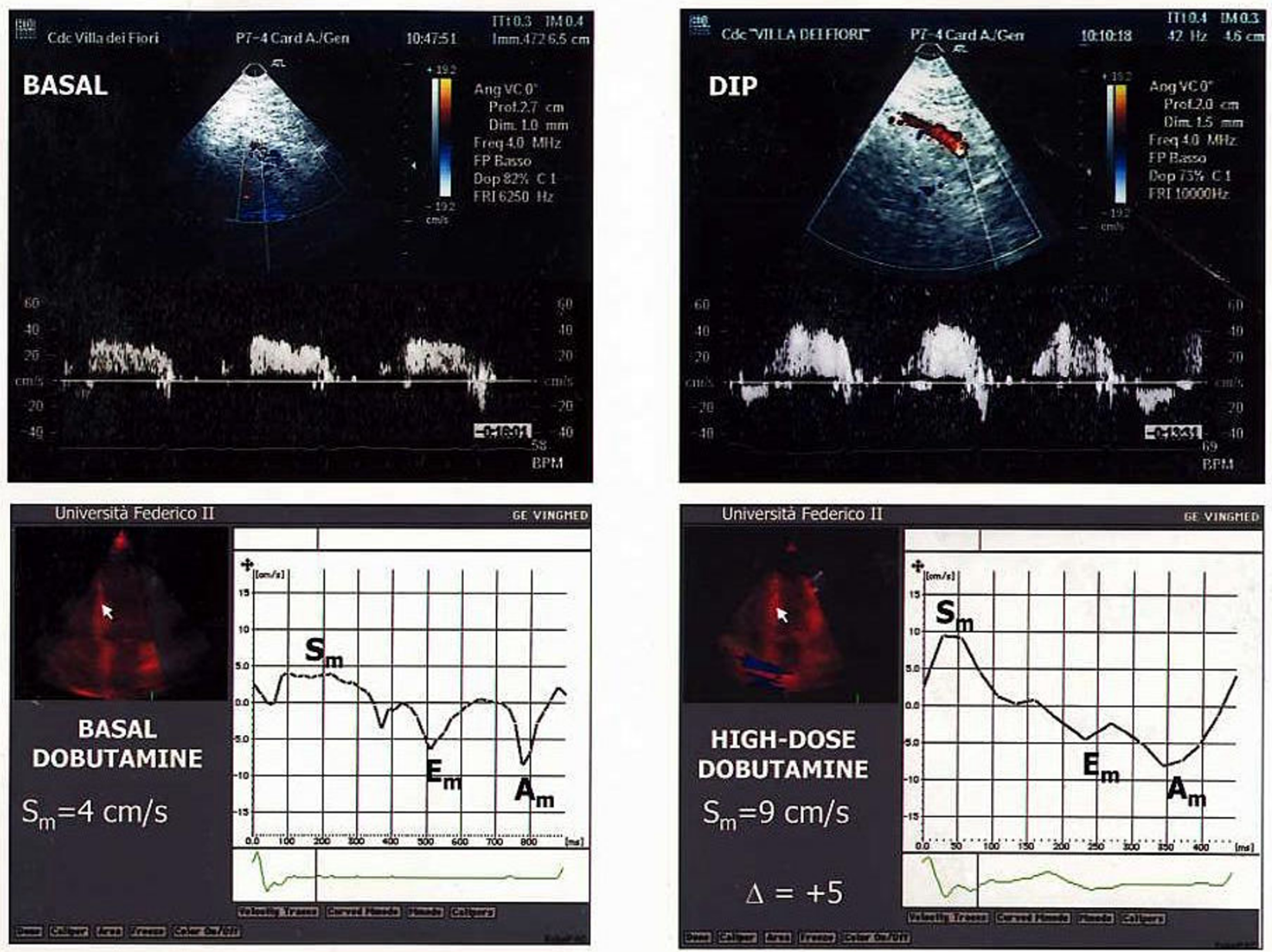

Figure 4

CFR and septal $S_{m}$ peak velocity at high-dose dobutamine in a patient with SPECT derived perfusion defect. The upper panels display a reduced CFR. In the lower panels, the increase of septal $S_{m}$ from baseline to high-dose dobutamine is low.

sibility that a residual coronary stenosis might be anatomically insignificant but hemodynamically important, thus explaining a discrepancy between the percentage of lumen reduction and the amount of regional flow reserve.

\section{Myocardial systolic function and perfusion defect after PTCA}

The reduction of myocardial systolic function expressed by the decrease of low and high-dose dobutamine $S_{m}$ in middle septum, i.e. in the territory supplied by LAD, is not surprising in patients with perfusion defects after PTCA. Of note, myocardial systolic performance of middle septum was not significantly different between the 2 groups at rest. These findings are consistent with an altered myo- cardial systolic velocity response to exercise already described by C-DT in patients with coronary artery disease $[13,41]$. Also WMSI, not different at rest, became significantly higher at low and high-dose dobutamine in patients with perfusion defects. This increase (involving LV segments of LAD territory) during stress occurred only in 5/11 patients who had lower $S_{m}$ peak velocities at peak dobutamine stress and lower CFR than patients without inducible wall motion abnormalities. Myocardial reperfusion injury may include LV regional systolic dysfunction as irreversible manifestation, it depending by a reduction of myocardial blood flow reserve [5]. Inducible wall motion abnormalities in the presence of a successful 
coronary revascularization might indicate a very severe microvascular damage.

Association between CFR and myocardial systolic function It is recognized that the extent of stress dobutamineinduced dissinergy is associated to the degree of CFR reduction in patients with significant coronary artery stenosis, an invasive myocardial fractional flow reserve $\leq 0.75$ having a sensitivity of $76 \%$ and a specificity of $97 \%$ [42]. In agreement with these findings, we found a positive association between the functional degree of vasodilator microvascular coronary circulation and the magnitude of regional myocardial systolic function at peak dobutamine stress, i.e. $\mathrm{S}_{\mathrm{m}}$ peak velocity of the wall (middle septum) supplied by LAD. Accordingly, we also found a lower but significant negative relation between CFR and stress peak WMSI. Since patients undergoing successful reperfusion procedures generally present a good stress-echo LV functional response [6], our data suggest the ability of C-DT to detect even minor forms of LV regional myocardial dysfunction occurring under these circumstances.

\section{Study Limitations}

The main limitation of the present study include the fact that the negativity of coronary angiography can not exclude definitely the presence of coronary restenosis while an invasive measurement of CFR by Doppler flow wire or an intra-coronary ultrasound could have been crucial to clarify this issue. Unfortunately, these evaluations were not included into our study protocol. In addition, it has also to be underscored that patients with perfusion defects of the present study had greater LV mass, a factor which can itself induce a reduction of CFR $[39,40]$.

\section{Clinical implications}

The results of the present study suggest that CFR impairment may be detectable after PTCA even in the absence of coronary restenosis, it depending by an altered coronary microvascular function. In this clinical scenario, SPECT stress perfusion defects have to be interpreted as false positive results for PCTA restenosis while they reflect a true physiologic impairment in regional CFR with some associated degree of systolic impairment detectable by C-TD. Quantitative parameters of CFR and C-TD can provide an additive value over conventional stress echocardiographic assessment.

\section{Competing interests Financial competing interests}

- In the past five years we didn't receive reimbursements, fees, funding, or salary from an organization that may in any way gain or lose financially from the publication of this manuscript, either now or in the future.
- No organization financied this manuscript.

- We didn't hold any stocks or shares in an organization that may in any way gain or lose financially from the publication of this manuscript, either now or in the future.

- We didn't hold or are currently applying for any patents relating to the content of the manuscript We didn't receive reimbursements, fees, funding, or salary from an organization that holds or has applied for patents relating to the content of the manuscript.

- We don't have any other financial competing interests.

\section{Non-financial competing interests}

There are not any non-financial competing interests (political, personal, religious, academic, intellectual, commercial or any other) to declare in relation to this manuscript.

We have not a competing interest, please discuss it with the editorial office.

\section{References}

I. RITA-2 trial participants: Coronary angioplasty versus medical therapy for angina: the second Randomised Intervention Treatment of Angina (RITA-2) trial. Lancet 1997, 350(9076):46|-468.

2. Erbel R, Haude M, Hopp HW, Franzen D, Rupprecght HJ, Heunlein B: Coronary-artery stenting compared with balloon angioplasty in patients with coronary artery disease: Benestent Study Group. N Engl J Med I 994, 33 I:489-495.

3. Topol E): Coronary artery stents: gauging, gorging and gouging. N Engl J Med 1998, 339: I702-1704.

4. Hansen PR: Myocardial reperfusion injury: experimental evidence and clinical relevance. Eur Heart J 1995, 16:734-740.

5. Van Liebergen RAM, Pick JJ, Koch KT, de Winter RJ, Lie KI: Immediate and long-term effect of balloon angioplasty or stenting implantation on the absolute and relative coronary blood flow velocity reserve. Circulation 1998, 98:2133-2140.

6. Bax JJ, Wijns W, Cornel JH, Visser FC, Boersma E, Fioretti PM: Accuracy of the currently available techniques for prediction of functional recovery after revascularization in patients with left ventricular dysfunction due to chronic coronary artery disease: comparison of pooled data. J Am Coll Cardiol 1997, 30:145I-I460.

7. Takeuchi M, Miura Y, Toyokawa T, Araki M, Nakashima Y, Kuroiwa A: The comparative diagnostic value of dobutamine stress echocardiography and thallium stress tomography for detecting restenosis after coronary angioplasty. I Am Soc Echocardiogr 1995, 8:696-702.

8. Heinle SK, Lieberman EB, Ancukiewicz M, Waugh RA, Bashore TM, Kisslo J: Usefulness of dobutamine echocardiography for detecting restenosis after percutaneous transluminal coronary angioplasty. Am J Cardiol 1993, 72: I220-I 225.

9. Beygui $F$, Le Feuvre C, Maunoury C, Helft G, Antonietti T, Metzger JP, Vacheron A: Detection of coronary restenosis by exercise electrocardiography thallium-20I perfusion imaging and coronary angiography in asymptomatic patients after percutaneous transluminal coronary angioplasty. Am J Cardiol 2000, 86:35-40.

10. Picano E, Bedetti G, Varga A, Cseh E: The comparable diagnostic accuracies of dobutamine-stress and dipyridamole-stress echocardiographies : a meta-analysis. Coron Artery Dis 2000, II:15I-159.

II. Caiati C, Montaldo C, Zedda N, Bina A, lliceto S: A new non-invasive method for coronary flow reserve assessment: contrast- 
enhanced transthoracic second harmonic echo Doppler. Circulation 1999, 99:77।-778.

12. Hozumi T, Yoshida K, Ogata Y, Akasaka T, Asami Y, Morioka S: Noninvasive assessment of significant left anterior descending coronary artery coronary stenosis by coronary flow velocity reserve with transthoracic color Doppler echocardiography. Circulation 1998, 97:| 557-I562.

13. Pasquet A, Armstrong G, Rimmerman C, Marwick TH: Correlation of myocardial Doppler velocity response to exercise with independent evidence of myocardial ischemia by dual-isotope single-photon emission computed tomography. $\mathrm{Am} \mathrm{J}$ Cardiol 2000, 85:536-542.

14. Galderisi M, Cicala S, De Simone L, Caso P, Petrocelli A, Pietropaolo L, Celentano A, Mininni N, de Divitiis O: Impact of myocardial diastolic dysfunction on coronary flow reserve in hypertensive patients with left ventricular hypertrophy. Ital Heart J 200I, 2:677-684.

15. Wilson RF, Johnson MR, Marcus ML, Aylward PE, Skorton DJ, Collins $S$, White CW: The effect of coronary angioplasty on coronary flow reserve. Circulation 1988, 77:873-885.

16. De Puey EG, Parmett S, Ghesani M, Rozanski A, Nichols K, Salensky $\mathrm{H}$ : Comparison of Tc-99m sestamibi and TI-20I gated perfusion SPECT. J Nucl Cardiol 1999, 6:278-285.

17. Bavelaar-Croon CD, Pauwels EK, van der Wass EE: Gated singlephoton emission computed tomographic myocardial imag ing: a new tool in clinical cardiology. Am Heart J 200I, | 41 1:383-390.

18. Sahn DJ, De Maria A, Kisslo J, Weyman A, for the Committee of MMode standardization of the American Society of Echocardiography: Recommendations regarding quantitation in M-Mode echocardiography: results of a survey of echocardiographic measurements. Circulation 1978, 58:1072-1083.

19. de Simone G, Daniels SR, Devereux RB, Meyer RA, Roman MJ, de Divitiis $\mathrm{O}$, Alderman $\mathrm{MH}$ : Left ventricular mass and body size in normotensive children and adults: assessment of allometric relations and impact of overweight. J Am Coll Cardiol 1992, 20: $125 \mid-1260$

20. Schiller NB, Shah PM, Crawdford M, De Maria A, Devereux RB, Feigenbaum $\mathrm{H}$, for the American Society of Echocardiography Committee on Standards, Subcommittee on Quantitation of Two-dimensional Echocardiograms: Recommendations for quantitation of the left ventricle by two-dimensional echocardiography. J Am Soc Echocardiogr 1989, 2:358-367.

21. Armstrong WF, Pellikka PA, Ryan T, Crouse L, Zoghbi WA: Stress echocardiography: recommendations for performance and interpretation of stress echocardiography. Stress Echocardiography Task Force of the Nomenclature and Standards Committee of the American Society of Echocardiography. Am Soc Echocardiogr 1998, II:97-104.

22. Galderisi M, Cicala S, Caso P, De Simone L, D'Errico A, Petrocelli A, de Divitiis $\mathrm{O}$ : Coronary flow reserve and myocardial diastolic dysfunction in arterial hypertension. Am J Cardiol 2002, 90:860-864.

23. Cannon RO: Restenosis after angioplasty. N Engl J Med 2002, 346: $1182-1183$.

24. Chilian WM, Layne SM: Coronary microvascular responses to reductions in perfusion pressure: evidence for persistent arteriolar vasomotor tone during coronary hypoperfusion. Circ Res 1990, 66:1227-1238.

25. Fragasso G, Lu C, Dabrowski P, Pagnotta P, Sheiban I, Chierchia SI: Comparison of stress/rest myocardial perfusion tomography, dipyridamole and dobutamine stress echocardiography for the detection of coronary disease in hypertensive patients with chest pain and positive exercise test. J Am Coll Cardiol 1999, 34:44I-447.

26. Hasdai D, Gibbons RJ, Holmes DR Jr, Higano ST, Lerman A: Coronary endothelial dysfunction in humans is associated with myocardial perfusion defects. Circulation 1997, 96:3390-95

27. Nanto S, Kodama K, Hori M, Mishima A, Inoue M, Kamada T: Temporal increase in resting coronary blood flow causes an impairment of coronary flow reserve after balloon angioplasty. Am Heart J 1992, I 23:28-36.

28. Kern MJ, Deligonul U, Vandormael M, Labovitz A, Gudipati CV, Gabliani G, Bodet J, Shah Y, Kennedy HL: Impaired coronary vasodilator reserve in the immediate post-coronary angioplasty period: analysis of coronary artery flow velocity indexes and regional cardiac venous efflux. I Am Coll Cardiol 1989, 13:860-872.

29. Miller DD, Verani MS: Current status of myocardial perfusion imaging after percutaneous transluminal coronary angioplasty. J Am Coll Cardiol 1994, 24:260-266.

30. Fischell TA, Bausbach KN, McDonald TV: Evidence of altered epicardial coronary artery autoregulation as a cause of distal coronary vasoconstriction after successful percutaneous transluminal coronary angioplasty. J Clin Investig 1990, 86:575-584.

31. Uren NG: Delayed recovery of coronary resistive vessel function after coronary angioplasty. I Am Coll Cardiol 1993, 2I:6|2-62I.

32. Zulstra F, Reiber JC, Julliere Y, Serruys PW: Normalization of coronary flow reserve by percutaneous transluminal coronary angioplasty. Am J Cardiol 1988, 61:55-60.

33. Albertal M, Regar E, Van Langenhove G, Carlier SG, Serrano P, Boersma E, Bruyne B, Di Mario C, Piek J, Serruys PW, on behalf of the DEBATE investigators: Flow velocity and predictors of suboptimal coronary flow velocity reserve after coronary balloon angioplasty. Eur Heart J 2002, 23:133-138.

34. Smith TP, Canty JM: Modulation of coronary autoregolatory responses by nitric oxide. Circ Res 1993, 73:232-240.

35. Marzilli M, Sambuceti G, Fedele S, L'Abbate A: Coronary microcirculatory vasoconstriction during ischemia in patients with unstable angina. J Am Coll Cardiol 2000, 35:327-334.

36. Pitkanen OP, Nuutila P, Raitakari OT, Ronnemaa T, Koskinen PJ, lida H, Lehtimaki T], Laine HK, Takala T, Viikari JS, Knuuti J: Coronary flow reserve is reduced in young men with IDDM. Diabetes 1998, 47:248-254.

37. Yokoyama I, Ohtake T, Momomura S, Yonekura K, Woo-Soo S, Nishikawa J, Sasaki Y, Omata M: Hyperglycemia rather than insulin resistance is related to reduced coronary flow reserve in NIDDM. Diabetes 1998, 47: I 19-124.

38. Astarita C, Palinkas A, Nicolini E, Maresca FS, Varga A, Picano E: Dypiridamole-atropine stress echocardiography versus exercise SPECT scintigraphy for detection of coronay artery disease hypertensive with positive exercise test. J Hypertens 2001, 19:679-682.

39. Gimelli A, Schneider-Eicke J, Neglia D, Sambuceti G, Giorgetti A, Bigalli G, Parodi G, Pedrinelli R, Parodi O: Homogeneously reduced versus regionally impaired myocardial blood flow in hypertensive patients: two different patterns of myocardial perfusion associated with degree of hypertrophy. J Am Coll Cardiol 1998, 31:366-373.

40. Hamasaki S, Al Suwaidi J, Higano ST, Miyauchi K, Holmes DR jr, Lerman A: Attenuated coronary flow reserve and vascular remodeling in patients with hypertension and left ventricular hypertrophy. J Am Coll Cardiol 2000, 35:1654-60.

41. Cain P, Baglin T, Case C, Spicer D, Short L, Marwick TH: Application of tissue Doppler to interpretation of dobutamine echocardiography and comparison with quantitative coronary angiography. Am / Cardiol 200I, 87:525-53I.

42. Bartunek J, Marwick TH, Rodrigues AC, Vincent M, Van Schuerbeeck $E$, Sys SU, de Bruyne B: Dobutamine-induced wall motion abnormalities: correlation with myocardial fractional flow reserve and quantitative coronary angiography. J Am Coll Cardiol 1996, 27:1429-1436.

Publish with BioMed Central and every scientist can read your work free of charge

"BioMed Central will be the most significant development for disseminating the results of biomedical research in our lifetime. "

Sir Paul Nurse, Cancer Research UK

Your research papers will be:

- available free of charge to the entire biomedical community

- peer reviewed and published immediately upon acceptance

- cited in PubMed and archived on PubMed Central

- yours - you keep the copyright
BioMedcentral 